\title{
Agreement Between Computed Tomography And Magnetic Resonance Imaging In Measuring Optic Nerve Sheath Diameter
}

\author{
Haider N. Al-Tameemi ${ }^{1} \&$ Neda M. Helel ${ }^{2}$ \\ ${ }^{1}$ Department of Surgery/Radiology Section, Faculty of Medicine, University of Kufa, Najaf, Iraq \\ ${ }^{2}$ Department of Radiology, Al-Sadder Medical City, Najaf, Iraq \\ Correspondence: Haider N. Al-Tameemi, Department of Surgery/Radiology Section, Faculty of Medicine, \\ University of Kufa, Najaf 54001, Iraq. Tel: 964-33-340-951. E-mail: haidern.altameemi@uokufa.edu.iq
}

\author{
Received: January 8, 2018 Accepted: February 20, 2018 Online Published: February 28, 2018 \\ doi:10.5539/gjhs.v10n4p22 URL: https://doi.org/10.5539/gjhs.v10n4p22
}

\begin{abstract}
Background: Neuroimaging is increasingly used as a non-invasive method to assess raised intracranial pressure (ICP). Optic nerve sheath diameter (ONSD) measurement using brain magnetic resonance imaging (MRI) has been shown to correlate well with invasively measured ICP, however little research has been conducted on the ONSD measurement using computerized tomography (CT) in correlation with ICP. This study was done to investigate whether $\mathrm{CT}$ scan can reliably replace MRI in measuring ONSD.

Method: A cross-sectional comparative study was conducted on 50 adult patients (29 females and 21 males), who underwent both CT and MRI of the brain along 10-month period. Using the brain axial section, the transverse ONSD was measured at $3 \mathrm{~mm}$ behind the globe in both modalities. Agreement between CT and MRI readings was assessed using intraclass correlation (ICC) and Kappa method.

Results: There was a strongly positive and statistically significant correlation between ONSD measurement using $\mathrm{CT}$ scan and MRI (p value $<0.001$ ). There was almost perfect agreement between CT scan and MRI in measuring ONSD (ICC $=0.987$ and Kappa $=0.837)$. Similar agreement was obtained when cases stratified into normal $(\leq 5 \mathrm{~mm})$ and thickened $(>5 \mathrm{~mm}$ ) ONSD (ICC $=0.947$ and 0.972 respectively).

Conclusion: CT scan is a reliable substitute for MRI in measuring ONSD with almost perfect agreement between the two modalities. It might be good practice to include ONSD measurement in the initial evaluation of brain CT scan in any patient with suspected raised ICP.
\end{abstract}

Keywords: magnetic resonance imaging, computerized tomography, optic nerve sheath, intracranial pressure

\section{Introduction}

\subsection{Intracranial Hypertension}

Intracranial hypertension defined as sustained ( $>5 \mathrm{~min}$ ) elevation of intracranial pressure (ICP) of $>20 \mathrm{mmHg}$ in adult (Susan et al., 2007) can result in serious neurological consequences if untreated. Therefore, early detection of raised ICP is critical to ensuring timely and appropriate management. Different causes of increased ICP have been reported including both intracranial (including head trauma, cerebral edema, hydrocephalus, hemorrhage, brain tumors (Saul \& Ducker, 1982; Bingaman \& Frank, 1995) and extracranial (hepatic encephalopathy) (Lidofsky et al., 1992). Direct ventriculostomy is the gold standard method for measuring ICP; however, this test is invasive and in addition to the technical difficulties carries risks of infection and hemorrhage (Rickert \& Sinson, 2003; Miller, 1987). To avoid these complications, noninvasive methods of measuring ICP were looked for, including transcranial Doppler ultrasonography and tympanic membrane displacement, however, they have so many technical difficulties, drawbacks and interobserver variability that preclude their clinical use (Bellner, Rommer, \& Reinstrup, 2004; Voulgaris, Parthenni, \& Kaliora, 2005; Shimbles, Dodd, \& Banister, 2005).

\subsection{Optic Nerve Sheath Diameter in Patients With Raised ICP}

Interest has been turned into the use of the optic nerve sheath diameter (ONSD) to provide a noninvasive surrogate marker for early-elevated ICP. This utilizes the fact that optic nerve with its CSF-filled sheath is anatomically considered part of the brain and when ICP is increased, the optic nerve sheath distends (Hansen \& Helmke, 1996) and this transmission of raised ICP into the optic nerve sheath has been recognized for many years (Geeraerts et al., 2008). Correlation has been found between ONSD and raised ICP (Hightower, Eric, \& Jason, 2012) and ultrasound (US) and magnetic resonance imaging (MRI) have been utilized to assess the ICP by measuring ONSD. US has 
been regarded as a simple bed side non-invasive technique to predict raised ICP with good accuracy (Kimberly \& Noble, 2008; Dubourg, Javouhey, Geeraerts, Messerer, \& Kassai, 2011)

Several studies conducted on MRI have proved its ability to predict raised ICP (Mashima et al., 1996; Weigel, Lagreze, Lazzaro, Hennig, \& Bley, 2006; Geeraerts et al., 2008; Kimberly \& Noble, 2008). Unlike MRI and US and up to our best knowledge, correlation between ICP and ONSD measured on brain CT scan was not thoroughly studied until recently (Kalantari, Jaiswal, Bruck, \& Matari, 2013; Mypinder, Donald, Chiara, \& Katherine, 2014). The purpose of this study is to assess the reliability of brain CT scan, a frequently utilized and available imaging modality as an alternative imaging tool to MRI in measuring ONSD.

\section{Method}

\subsection{Participants}

Adult (18-year and older) patients who had both CT and MRI brain examinations but without history of ophthalmic disease, orbital trauma or orbital surgery and no evidence of orbital pathology.

\subsection{Sampling Procedure}

Patients were sampled from those referred to the CT/MRI unit during a 10-month period for different indications (different medical conditions like headache, fit, stroke and tumors). Only patients who had both MRI and CT examinations of the brain done at the same visit (same day) within short interval were included in the study.

\subsection{CT and MRI Examinations}

\subsubsection{CT Scan}

All patients were examined by the same CT scanner (64 multidetectors helical, Brilliance, Philips Medical System, 2010) using the following parameters: KV 120, MAS 300mAs, slice thickness $0.9 \mathrm{~mm}$ and time 12 seconds. Patient in supine position, head in head rest, scanner gantry parallel to radiographic baseline. Radiological analysis was done using the brain axial sections.

\subsubsection{MRI}

All patients were examined by the same MRI device (1.5 Tesla device, Acheiva, Philips Medical System, 2011) with the following protocol; axial T2-weighted fast spin echo (FSE) images: TR $3500 \mathrm{msec}$, TE $100 \mathrm{msec}$, flip angle 90 , slice thickness $3.5 \mathrm{~mm}$, slice gap $0.3 \mathrm{~mm}$, matrix $320 \times 224$, field of view $16 \times 16 \mathrm{~cm}$, echo train length 12 , and bandwidth $31.2 \mathrm{kHz}$.

\subsection{Image Analysis and ONSD Measurement}

All scans were reviewed initially by a board-certified neuroradiologist for any abnormalities. ONSD measurements were performed manually by another observer using electronic calipers on workstation monitor (extended brilliant workstation). The transverse ONSD on both right and left sides was measured at $3 \mathrm{~mm}$ behind the globe in both modalities (Figures 1 and 2) using the axial sections. To avoid interobserver bias, the same observer measured ONSD on both CT and MRI images and to ensure blinding, measurements were done separately in different occasions ( $>2$ weeks interval) after "zooming in" the image on the orbits (to hid brain findings) and removing image information by another researcher.

We noticed that any modification in the window and center levels of the CT images would have obvious effects on the margins of optic nerve and consequently result in different measurements. Therefore, we ensured that all ONSD measurements on CT images were taken using the same values of brain window (80) and center (40) that are routinely utilized in the brain protocol of CT device in our institution.

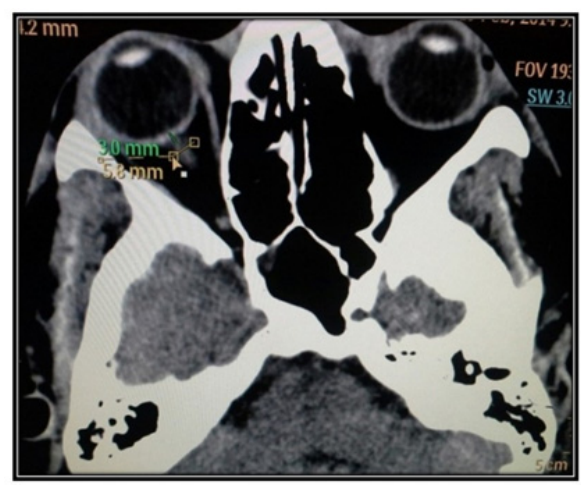

Figure 1. Measurement of optic nerve sheath diameter (ONSD) using computerized tomography (CT) scan. Axial image of the brain with right ONSD of $5.8 \mathrm{~mm}$ measured at $3 \mathrm{~mm}$ behind the globe (window 80 and center 40) 


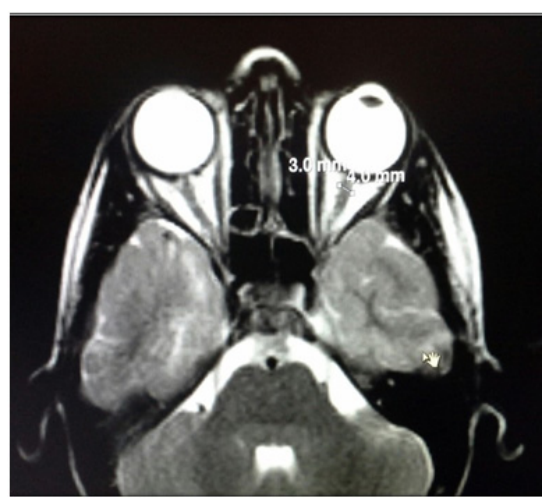

Figure 2. Measurements of optic nerve sheath diameter (ONSD) by magnetic resonance imaging (MRI). Axial T2-weighted MR image of the brain with left ONSD of 4mm measured at $3 \mathrm{~mm}$ behind the globe

\subsection{Statistical Analysis}

Measurements of ONSD, including right-sided, left-sided and the means of both were compared between the two modalities. Statistical analysis was done using SPSS (version 2.0.) We considered $0.2 \mathrm{~mm}$ as an acceptable difference between the CT and MRI scans in ONSD measurement as it is the lowest value of the reported discrepancy range ( 0.2 to $0.7 \mathrm{~mm}$ ) (Soldatos et al., 2008; , Moretti, Pizzi, Cassini \& Vivaldi, 2009). The correlation between ONSD on CT and MRI was calculated with $95 \%$ confidence interval and a p-value $<0.05$ was set as significant. The agreement between CT with MRI in measuring ONSD was assessed using intra-class correlation (ICC) between means of ONSD on both modalities. ICC can be interpreted as follows: $0-0.2=$ poor agreement; 0.3-0.4 =fair agreement; 0.5-0.6 = moderate agreement; $0.7-0.8=$ strong agreement; and $>0.8=$ almost perfect agreement (Gisev, Bell, \& Chen, 2013).

Furthermore, categorizing the data with a $5 \mathrm{~mm}$ as cut-off limit, we used Kappa statistics to assess the agreement between CT and MRI in measuring normal $(\leq 5 \mathrm{~mm})$ and thickened $(>5 \mathrm{~mm})$ ONSD. Interpretation of Kappa measurements was as follows: poor agreement $<0.20$, fair agreement $=0.20-0.39$; moderate agreement $=0.40-0.59$; good (substantial) agreement $=0.60-0.79 ;$ and very good $($ perfect $)$ agreement $=0.80-1.00($ Altman, 1991) .

The institutional review board has approved the study. There was no clinical intervention in this study, all patients gave informed consent prior to their inclusion in the study and confidentiality of personal data is preserved.

\subsection{Study Design}

This is a cross-sectional, observational comparative study conducted on 50 patients ( 29 female and 21 male). No intervention was performed.

\section{Results}

\subsection{Basic Demographic Data}

The study included 50 patients and their characteristics are demonstrated in Table 1.

Table 1. Demographic data of the 50 patients included in the study

\begin{tabular}{ll}
\hline Parameter & Values \\
\hline Age (years) & $39.8 \pm 15.8$ \\
Gender & 21 \\
Male & 29 \\
Female & $89.3 \pm 10.7$ \\
Weight (kg) & $18(36 \%)$ \\
Brain radiological findings & $19(38 \%)$ \\
Normal & $7(14 \%)$ \\
SOL &
\end{tabular}




$\begin{array}{ll}\text { Hemorrhage } & 5(10 \%) \\ \text { Hydrocephalus } & 1(2 \%) \\ \text { Mean ONSD }^{* *}(\mathrm{~mm}) & \\ \mathrm{CT}^{* * *} & 4.792 \pm 1.003 \\ \mathrm{MR}^{\mathrm{I***}} & 4.937 \pm 1.025\end{array}$

*SOL=space occupying lesion; $* * \mathrm{ONSD}=$ optic nerve sheath diameter $* * * \mathrm{CT}=$ computerized tomography; $* * * * \mathrm{MRI}=$ magnetic resonance imaging.

\subsection{Main Findings}

Strongly positive and statistically significant correlation ( $p$ value $<0.001$ ) was found between measurements of ONSD using CT scan and MRI (Figure 3).

The agreement between means of ONSD measured on MRI with those measured on CT scan in all cases was almost perfect ( ICC $=0.987$, p value $<0.001$ ) (Table 2 ) with almost all readings were distributed tightly around the midline of the acceptable difference of $0.2 \mathrm{~mm}$ (Figure 4) indicating that there is almost no variation between the two modalities.

Similarly, when cases were stratified according to the ONSD measurements into those with normal $(\leq 5 \mathrm{~mm})$ and those with thickened $(>5 \mathrm{~mm}$ ) ONSD, the agreement was also almost perfect (ICC $=0.947$ and 0.972 respectively) (Table 2) with similar tight clustering of ONSD readings around the acceptable difference of $0.2 \mathrm{~mm}$ (Figures 5 and 6 respectively).

Table 2. Agreement between CT scan and MRI in ONSD measurement

\begin{tabular}{|c|c|c|c|c|}
\hline & Mean *ONSD & Std. Deviation & Intraclass correlation & $\mathrm{P}$ value \\
\hline \multicolumn{5}{|c|}{ All ONSD } \\
\hline$* * \mathrm{CT}$ & 4.7920 & 1.00277 & \multirow{2}{*}{0.987} & \multirow{2}{*}{$<0.001$} \\
\hline$* * * \mathrm{MRI}$ & 4.9370 & 1.02528 & & \\
\hline \multicolumn{5}{|c|}{ ONSD $\leq 5 \mathrm{~mm}$} \\
\hline $\mathrm{CT}$ & 4.0370 & 0.46006 & \multirow{2}{*}{0.972} & \multirow{2}{*}{$<0.001$} \\
\hline MRI & 4.1352 & 0.46323 & & \\
\hline \multicolumn{5}{|c|}{ ONSD $>5 \mathrm{~mm}$} \\
\hline CT & 5.6783 & 0.68471 & \multirow{2}{*}{0.947} & \multirow{2}{*}{$<0.001$} \\
\hline MRI & 5.8783 & 0.61030 & & \\
\hline
\end{tabular}

$* \mathrm{ONSD}=$ optic nerve sheath diameter ${ }^{* *} \mathrm{CT}=$ computerized tomography $; * * * \mathrm{MRI}=$ magnetic resonance imaging. 


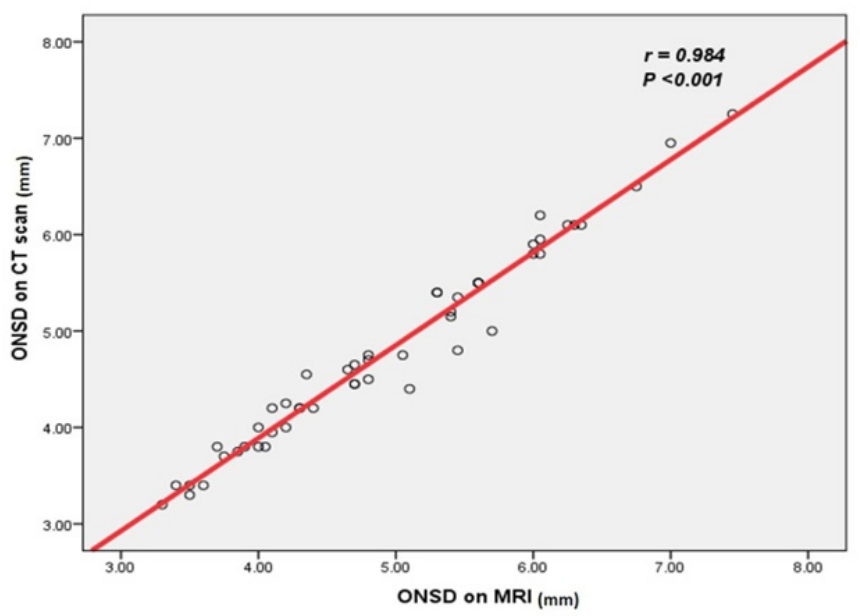

Figure 3. Correlation between optic nerve sheath diameters (ONSD) measured on computerized tomography (CT) and magnetic resonance imaging (MRI)

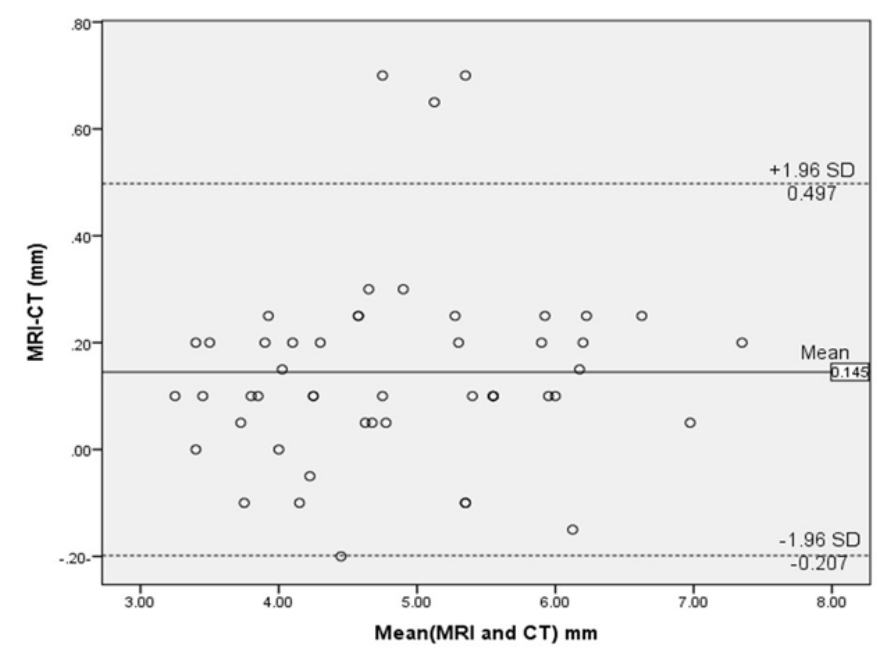

Figure 4. Agreement between computerized tomography (CT) and magnetic resonance imaging (MRI) in all optic nerve sheath diameter (ONSD) measurements

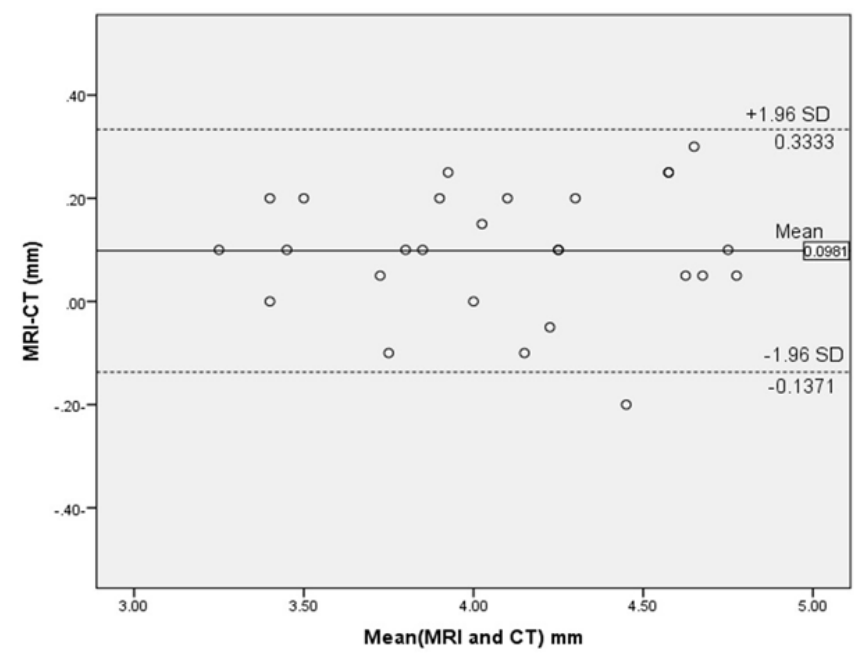

Figure 5. Agreement between computerized tomography (CT) and magnetic resonance imaging (MRI) when optic nerve sheath diameter (ONSD) $\leq 5 \mathrm{~mm}$ 


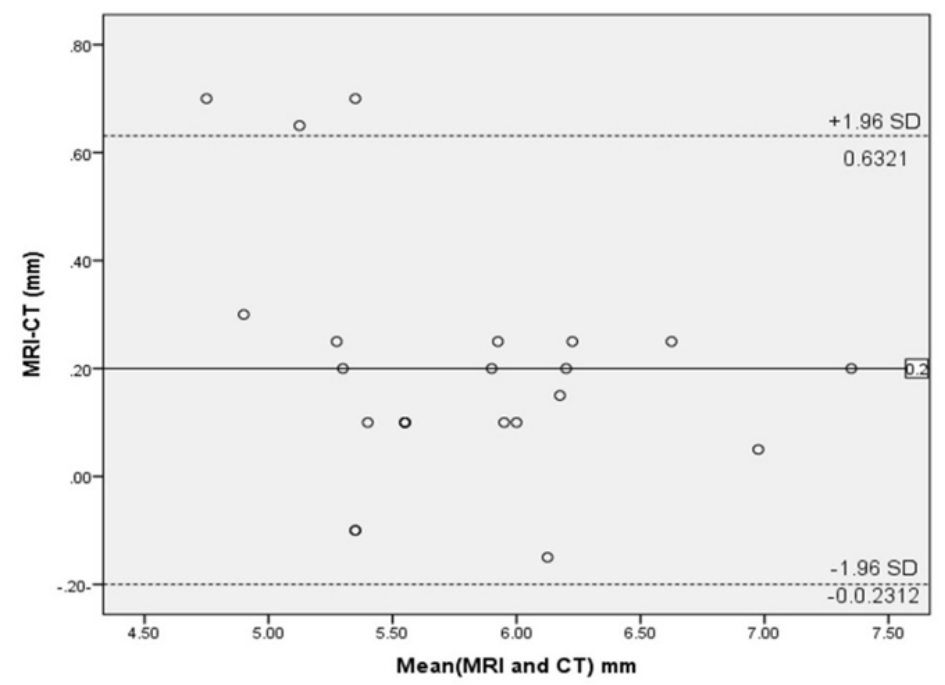

Figure 6. Agreement between computerized tomography (CT) and magnetic resonance imaging (MRI) when optic nerve sheath diameter $(\mathrm{ONSD})>5 \mathrm{~mm}$

\subsection{Additional Statistical Analysis}

To consolidate our results, we assessed the overall agreement between all readings using Kappa method on categorical data i.e. ONSD $\leq 5 \mathrm{~mm}$ and those $>5 \mathrm{~mm}$. There was almost perfect agreement (Kappa 0.837) between measurements of ONSD by CT scan and MRI as seen in Table 3.

Table 3. Kappa agreement between CT and MRI in measuring ONSD

\begin{tabular}{lllll}
\hline & & \multicolumn{2}{l}{ ONSD using CT*** scan } & Total \\
\cline { 3 - 5 } & & $\leq 5 \mathrm{~mm}$ & $>5 \mathrm{~mm}$ & 27 \\
\hline ONSD* & & 27 & 0 & 23 \\
MRI** & & 4 & 19 & 50 \\
Total & & 31 & 19 & $5 \mathrm{~mm}$ \\
\hline
\end{tabular}

*ONSD $=$ optic nerve sheath diameter; $* * \mathrm{MRI}=$ magnetic resonance imaging; ${ }^{* * *} \mathrm{CT}=$ computerized tomography.

\section{Discussion}

Being a safe and non-invasive tool compared to more risky invasive methods, ONSD measurement is increasingly utilized in the evaluation of patients with or suspected to have raised ICP using US or MRI.

Although MRI was shown to be a reliable surrogate for the directly derived ICP ((Kimberly \& Noble, 2008; Geeraerts et al., 2008), CT scan was not evaluated comprehensively to be considered similarly reliable. One of the early studies addressing this issue found a strong correlation between CT-derived ONSD and invasively-measured ICP (Mypinder, Donald, Chiara, \& Katherine, 2014). The strong and statistically significant correlation between CT- and MRI-derived ONSD readings obtained in our study is evidence that CT scan can be as reliable as MRI. Mypinder et al (2014) also found that measuring ONSD on CT scan has resulted in a more accurate prediction of raised ICP than other reported signs of raised ICP in brain CT scan like ventricular and sulcal effacement. This correlation between CT and MRI was also consistent with a research by Kalanteri et al. (2013). However, our study differs from Kalanteri et al. in that all cases included in their comparison were of normal ONSD range (taking $5 \mathrm{~mm}$ as a normal cut-off value) (Ossoinig, 1993; Geeraerts et al., 2008; Kimberly \& Noble, 2008), while cases with both normal and thickened ONSD were included in our study.

The agreement was almost perfect when taking all CT and MRI readings with slightly higher ICC than Kalanteri et al. (0.987 versus 0.89 respectively) indicating a lesser variation and better concordance between the measurements.

Because Kalanteri et al studied only the ONSD of normal limit $(\leq 5 \mathrm{~mm})$, comparability of CT to MRI in abnormal 
(thickened) ONSD might be questionable. Therefore, we assessed the agreement between CT and MRI using ICC in cases with normal $(\leq 5 \mathrm{~mm})$ and abnormal $(>5 \mathrm{~mm})$ ONSD and it was interestingly almost perfect in both subgroups. To our best knowledge, no published research has evaluated this agreement (between abnormal ONSD measurements on CT and MRI) to compare it with our results. Although more research work may be still necessary, our preliminary results indicate that CT would be a reliable alternative to MRI not only in normal ONSD but also in abnormal (thickened) ONSD.

Kappa method is usually used to assess the agreement between two modalities or observers but with categorical data. Therefore we used it to compare ability of CT to agree with MRI in determining whether ONSD is normal or abnormal, setting $5 \mathrm{~mm}$ as a cut-off normal limit. Similarly, we found almost perfect (kappa $=0.837$ ) agreement between both modalities, hence statistically consolidating the capability of CT in replacing MRI in the assessment of ONSD. Compared to MRI, CT scan is more readily available, especially in emergency situations, less expensive, faster and relatively safer for patients who have contraindications to MRI, particularly traumatic patients with resuscitation/support devices, irritability and/or foreign bodies. Therefore, $\mathrm{CT}$ scan would be a reasonable solution in such critical conditions when prediction of raised ICP is required.

This study has some limitations. First is related to MR images used for ONSD measurement as we measured ONSD on the fat-saturated and not fat-suppressed T2-weighted sequence already used in the routine brain protocol. Although fat-suppressed images may be more accurate for ONSD measurement, our results using non-fat suppressed images were encouraging. Secondly, ICP was not measured directly in our patients and we think that this might have added more to the study if it has been done. However the main purpose of the study was not to prove the correlation between ONSD and ICP but rather to assess if CT can replace MRI in particular task; that is measuring ONSD.

In conclusion, almost perfect agreement is present between CT and MRI in measuring ONSD. Therefore, CT scan can be considered as a reliable substitute for MRI in this task when assessment of raised ICP is required, especially where MRI is not available or contraindicated and in emergency or un-cooperative patients. It may also be beneficial to routinely include ONSD measurement in the initial evaluation of brain CT scans particularly in patients suspected of having raised ICP. Nevertheless, more studies are necessary to evaluate the correlation between CT-derived ONSD and the directly measured ICP.

\section{Competing Interests Statement}

The authors declare that there are no competing or potential conflicts of interest.

\section{References}

Altman, D. G. (1991). Practical Statistics for Medical Research (p. 404). London, England: Chapman and Hall.

Ballmer, J., Roomer, B., \& Reinstrup, P. (2004). Transcranial Doppler sonography pulsatility index (PI) reflects intracranial pressure (ICP). Surgical Neurology, 62, 45-51. https://doi.org/10.1016/j.surneu.2003.12.007

Bingaman, W. E., \& Frank, J. I. (1995). Malignant cerebral edema and intracranial hypertension. NeurolClin. Aug., 13(3), 479-509.

Bratton, S. L., Chestnut, R. M., Ghajar, J., McConnell Hammond, F., Harris, O. A., Hartl, R., .... Wright, D. W. (2007). Guidelines for the management of severe traumatic brain injury. VIII. Intracranial pressure thresholds. J Neurotrauma, 24 (Suppl 1), S55-8.https://doi.org/10.1089/neu.2007.9988

Dubourg, J., Javouhey, E., Geeraerts, T., Messerer, M., \&Kassai, B. (2011). Ultrasonography of optic nerve sheath diameter for detection of raised intracranial pressure: a systematic review and meta-analysis. Intensive Care, 37(7), 1059-1068. https://doi.org/10.1007/s00134-011-2224-2

Geeraerts, T., Newcombe, V., Coles, J., Abate, M., Perkes, I., Hutchinson, P., ... Menon, D. (2008). Use of T2-weighted magnetic resonance imaging of the optic nerve sheath to detect raised intracranial pressure. Crit Care, 12, R114. https://doi.org/10.1186/cc7006

Gisev, N., Bell, J. S., \& Chen, T. F. (2013). Interrater agreement and interrater reliability: key concepts, approaches, and applications. Res Social Adm Pharm., 9(3), 330-8. https://10.1016/j.sapharm.2012.04.004

Hansen, H., \& Helmke, K. (1996).The subarachnoid space surrounding the optic nerves. An ultrasound study of the optic nerve sheath. Surg. Radiol. Anat., 18, 323-328. https://doi.org/10.1007/BF01627611

Hightower, S., Eric, J., \& Jason, D. (2012).Detection of increased intracranial pressure by Ultrasound. Journal of Special Operations, 3(12), 19-22.

Kalantari, H., Jaiswal, R., Bruck, I., \& Matari, H. (2013). Correlation of optic nerve sheath diameter measurements 
by computed tomography and magnetic resonance imaging. American Journal of Emergency Medicine, 31, 1595-1597. https://doi.org/10.1016/j.ajem.2013.07.028

Kimberly, H., \& Noble, V. (2008).Using MRI of the optic nerve sheath to detect elevated intracranial pressure. Crit Care, 12, 181. https://doi.org/10.1186/cc7008

Lidofsky, S. D., Bass, N. M., Prager, M. C., Washington, D. E., Read, A. E., Wright, T. L., \& Lake, J. R. (1992). Intracranial pressure monitoring and liver transplantation for fulminant hepatic failure. Hepatology, 16(1), 1-7. https://doi.org/10.1002/hep.1840160102

Lundberg, N. (1960). Continuous recording and control of ventricular fluid pressure in neurosurgical practice, ActaPsychiatricaScandinavicaSupplementum, 36, 1-193.

Mashima, Y., Oshitari, K., Imamura, Y., Momoshima, S., Shiga, H., \& Oguchi, Y. (1996). High-resolution magnetic resonance imaging of the intraorbital optic nerve and subarachnoid space in patients with papilledema and optic atrophy. Arch Ophthalmol, 114, 1197-1203. https://doi.org/10.1001/archopht.1996.01100140397006

Miller, J. D. (1987). ICP monitoring - current status and future directions. Acta Neurochir (Wien), 85, 80-86. https://doi.org/10.1007/BF01456102

Moretti, R., Pizzi, B., Cassini, F., \& Vivaldi, N. (2009). Reliability of optic nerve ultrasound for the evaluation of patients with spontaneous intracranial hemorrhage. Neurocrit Care, 11, 406-410. https://doi.org/10.1007/s12028-009-9250-8

Mypinder, S., Donald, E., Chiara, R., \& Katherine, W. (2014). Optic nerve sheath diameter on computed tomography is correlated with simultaneously measured intracranial pressure in patients with severe traumatic brain injury. Intensive Care Medicine, 40(9), 1267-74. https://doi.org/10.1007/s00134-014-3392-7

Ossoinig, K. C. (1993). Standardized echography of the optic nerve. Doc Ophthalmol Proc Ser, 55, 3-99. https://doi.org/10.1007/978-94-011-1846-0_1

Rickert, K., \& Sinson, G. (2003). Intracranial pressure monitoring. Oper Tech Gen Surg, 5, 170-175. https://doi.org/10.1016/S1524-153X(03)70010-4

Saul, T. G., \& Ducker, T. B. (1982). Effect of intracranial pressure monitoring and aggressive treatment on mortality in severe head injury. J Neurosurg, 56(4), 498-503.

Shimbles, S., Dodd, C., \& Banister, K. (2005). Clinical comparison of tympanic membrane displacement with invasive intracranial pressure measurements. Physiological Measurement, 26(6), 1085-1092. https://doi.org/10.1088/0967-3334/26/6/017

Soldatos, T., Karakitsos, D., Chatzimichail, K., Papathanasiou, M., Gouliamos, A., \& Karabanis, A. (2008). Optic nerve sonography in the diagnostic evaluation of adult brain injury. Crit Care, $12,67$. https://doi.org/10.1186/cc6897

Voulgaris, S., Parthenni, M., \& Kaliora, H. (2005). Early cerebral monitoring using the transcranial Doppler pulsatility index in patients with severe brain trauma.Medical Science Monitor, 11(2), 49-52.

Weigel, M., Lagreze, W., Lazzaro, A., Hennig, J., \& Bley, T. (2006). Fast and quantitative high-resolution magnetic resonance imaging of the optic nerve at 3.0 tesla. Invest Radiol., 41, 83-86. https://doi.org/10.1097/01.rli.0000195820.98062.c5

\section{Copyrights}

Copyright for this article is retained by the author(s), with first publication rights granted to the journal.

This is an open-access article distributed under the terms and conditions of the Creative Commons Attribution license (http://creativecommons.org/licenses/by/4.0/). 